\title{
HAP1 positive control mutants specific for one of two binding sites
}

\author{
Bernard Turcotte and Leonard Guarente \\ Department of Biology, Massachusetts Institute of Technology, Cambridge, Massachusetts 02139 USA
}

\begin{abstract}
The expression of the yeast $C Y C 1$ and $C Y C 7$ genes is controlled by the HAP1 activator. A GAL4-like zinc finger (residues 1-148) specifies binding to the dissimilar sites UAS1 (of CYC1) and CYC7, and an acidic domain (residues 1307-1483) is essential for activation of transcription. To analyze how HAP1 binds to UAS1 and CYC7, we performed saturation mutagenesis of the DNA-binding domain and recovered mutants with altered activity. Class 1 mutants had a reduced activity at both UAS1 and CYC7, and class 2 mutants selectively eliminated activity at CYC7. Surprisingly, several mutants of both classes exhibited wild-type DNA binding, indicating that they were specifically defective in activation. These positive control (PC) mutants alter residues that bracket the zinc finger. We explain these mutants in a model involving cofactor proteins that bind UAS1 and CYC7 along with HAP1. The existence of PC mutants that only affect activity at CYC7 raises the possibility that different cofactors may exist for UAS1 and CYC7.
\end{abstract}

[Key Words: Yeast; transcription; HAP1; positive control mutants; cofactor; GAL11]

Received May 18, 1992; revised version accepted July 17, 1992.

Eukaryotic enhancers are sites found at locations distant from transcription initiation sites, which augment transcription (Banerii et al. 1981; Moreau et al. 1981). The activity of enhancers depends on transcriptional activator proteins that bind to the sites. Activators contain two functional domains: one that dictates site-specific DNA binding to the enhancer, and one dedicated to the activation of the transcriptional machinery (Brent and Ptashne 1985). Activation domains fall into several classes, the most prevalent of which contains a high concentration of acidic amino acids (Hope and Struhl 1986; $\mathrm{Ma}$ and Ptashne 1987). These acidic domains are functional in eukaryotes that range from yeast to mammals, an indication of striking conservation (for review, see Guarente and Bermingham-McDonogh 1992).

The enhancer and the initiation site-proximal promoter element, the TATA box, can be separated by hundreds of nucleotides, creating the capacity for complex arrays of enhancers and activators at a single promoter. In addition to the complexity of a multitude of activators acting at a single promoter, the existence of additional factors, or cofactors, can influence the potency of enhancers. For example, binding of the yeast mating-type regulators $\alpha 1$ and $\alpha 2$ to their target sites is aided by the binding of MCM1 to adjacent DNA sequences (Keleher et al. 1988; Jarvis et al. 1989; Passmore et al. 1989l. In the yeast $G A L$ system, the product of GAL11 potentiates full activity of the GAL4 activator (Suzuki et al. 1988) and shows specificity for the DNA-binding domain of GAL4 (Himmelfarb et al. 1990). This GAL4 domain contains a zinc finger of 6 cysteine residues that is very similar to DNA-binding domains of a number of yeast activators.
The activity of one of these activators, PPR 1 , is reduced in a gal11 mutant, suggesting that GAL11 may be a cofactor for this family of yeast activators (Himmelfarb et al. 1990).

Another yeast activator with similarity to GAL4 throughout the zinc finger domain is HAPl (Pfeifer et al. 1989). When compared with the other members of this family, HAP1 contains a longer amino-terminal extension adjacent to the finger. For example, the first cysteine residue of GAL4 is at residue 11, whereas in HAP1 the corresponding residue is at position 64 (Pfeifer et al. 1989). HAPl activates transcription of the two genes encoding cytochrome c, CYC1 and CYC7 (Guarente et al. 1984; Prezant et al. 1987; Zitomer et al. 1987), as well as genes encoding other heme-related proteins (Winkler et al. 1988; Schneider and Guarente 1991). The DNA-binding activity of HAP1 itself is stimulated by heme, which, presumably, binds to an internal sequence in the protein that otherwise blocks DNA binding. The structure of HAP1 contains discrete domains. The DNA-binding domain lies between residues 1 and 148, the heme regulatory domain is between residues 245 and 444, and the acidic activation domain is between residues 1307 and 1483 (Pfeifer et al. 1989).

Strikingly, while HAP1-binding sites at UAS1 (uppstream activating sequence 1 ) of $C Y C 1, C Y T 1$, and $C T T 1$ are similar, the $\mathrm{CY} C 7$ site shows no discernible similarity. Several findings suggest that the same HAP1 DNAbinding domain binds to the dissimilar sequences, UAS1 and CYC7. First, the sites cross-compete for HAP1 binding with an equal affinity (Pfeifer et al. 1987b). Second, mutations in the DNA-binding domain, including mu- 
tations in the Cys residues, knock out binding to both sites (Pfeifer et al. 1989). Gel-shift complexes of HAP1 or truncated derivatives to UAS1 and CYC7 show identical mobilities, suggesting that the same species of the protein binds to the sites. In addition, when the first 148 amino acids of HAPl were translated in vitro, the polypeptide bound to both UASl and CYC7 (Pfeifer et al. 1989).

What biological role might be served by multisequence recognition? Perhaps the most striking feature of the dissimilar HAP1 sites is that transcriptional activation by UAS1 and CYC7 differs quantitatively and qualitatively in vivo. Thus, when synthetic UAS1 and CYC7 oligonucleotides are inserted into the same promoter construct, the level of activation driven by HAP1 is 5- to 10-fold higher for UAS1 (Kim and Guarente 1989). Furthermore, while the activity of HAPl at UAS1 increases in cells shifted from glucose to lactate, the activity of HAPl at CYC7 decreases (L. Guarente, unpubl.).

An additional clue regarding the differential activity of UAS1 and CYC7 comes from the HAP1-18 mutation (Creusot et al. 1988; Pfeifer et al. 1989). This mutation was originally identified because it increases the activity of HAP1 at CYC7 10- to 100-fold (Clavilier et al. 1976). We found that the HAP1-18 mutation also increased the activity of HAP1 dramatically at the synthetic CYC7 oligonucleotide in front of a minimal CYC1 promoter (Kim and Guarente 1989). The mutation changes Ser-63 to Arg, immediately adjacent to the Cys cluster in the DNA-binding domain of the protein. A secondary effect of the change is that the mutant does not bind to UAS1. When Ser-63 is systematically changed to 15 other residues, only Arg-63 shows elevated activation of CYC7, although several other changes, like HAP1-18, selectively knock out binding to UAS1 (Kim and Guarente 1989).

Several models are consistent with these findings. We have suggested that the DNA sequence itself may alter the conformation of the DNA-binding domain to allow that part of HAPl to contact the transcriptional machinery differently when bound at the two sites (Kim and Guarente 1989). To begin to address the question of how HAPl function might differ at UAS1 versus CYC7, we have performed a systematic genetic analysis of the DNA-binding domain of HAP1 described herein. In so doing, we have isolated mutations that affect the activities of UAS1 and CYC7 differentially. A novel class of mutants retains the ability to bind to both sites but has lost the ability to activate specifically at one. From these results, we suggest that HAP1 activity in vivo is due partly to cofactor proteins that bind to CYC7 and UAS1. Our results raise the possibility that a single activator can interact with two different cofactors when bound at different sequence elements.

\section{Results}

\section{Genetic strategy}

We wished to carry out a saturation mutagenesis of the DNA-binding domain of HAP1 and then screen for mutations that differentially affected UAS1 (of CYC1) and the CYC7 element. To facilitate the mutagenesis, we constructed a plasmid that bore the HAP1 DNA-binding domain on a cassette so that it could be transferred from the substrate for mutagenesis to a yeast expression plasmid. The DNA-binding domain of HAPl was transferred to a plasmid with an M13 origin, and single-stranded DNA was prepared. Saturation mutagenesis of codons 48-130 was carried out using nine overlapping oligonucleotides (Fig. 1), which were synthesized such that they contained, on average, one mutation per oligonucleotide. Each synthetic oligonucleotide was extended using the single-stranded substrate, and resulting plasmids were transformed into Escherichia coli. Greater than $10^{4}$ in-

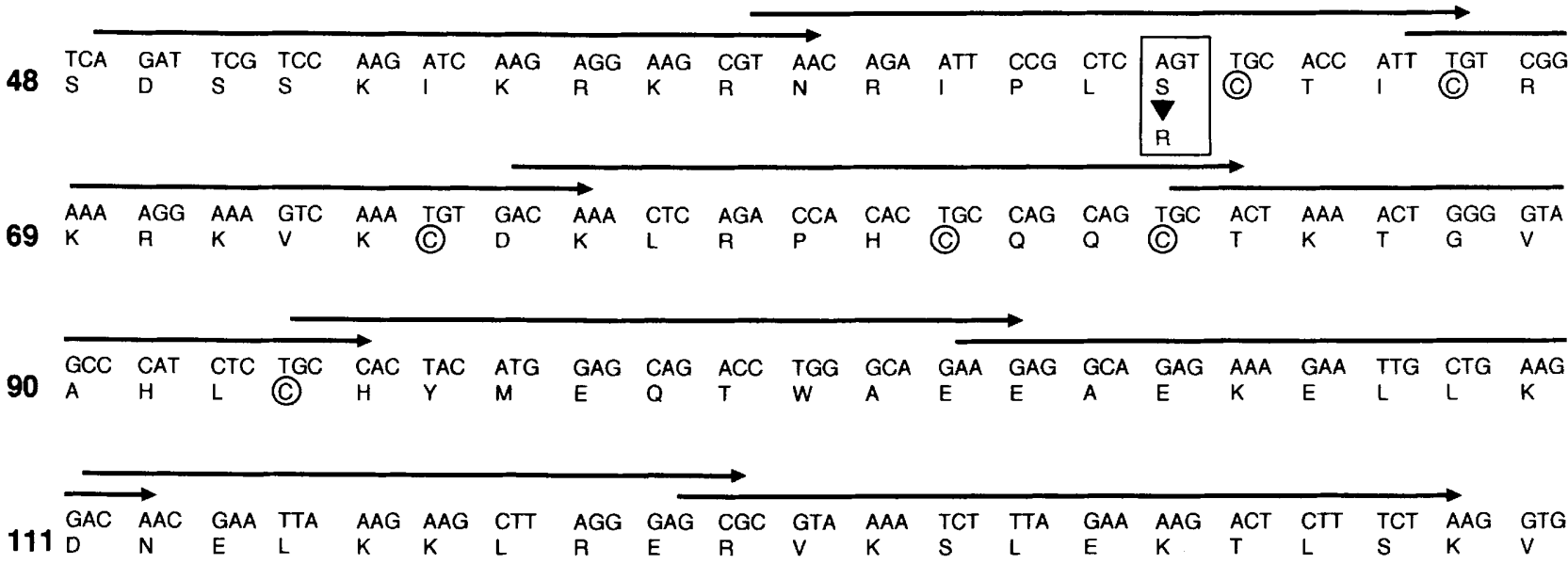

Figure 1. Nucleotide and amino acid sequence of the DNA-binding domain of HAP1. Amino acid numbering is given at left. Circled amino acids are cysteine residues of the zinc finger involved in binding to zinc. The rectangle shows the position of the HAP1-18 mutation. Arrows indicate the sequence of the spiked oligonucleotides that were used for saturation mutagenesis (see Materials and methods). 
dependent colonies were pooled, and the cassette was transferred to a yeast expression plasmid under control of the $\mathrm{UAS}_{G A L}$.

To screen for mutations that affected UAS1 and CYC7 differentially, the yeast strain LPY22 (Uhap1) was transformed with mutagenized HAP1 plasmid. The strain also contained a lacZ reporter driven by the CYC1 TATA box-mRNA initiation region and either UAS1 or CYC7 UAS. Both UAS1 and CYC7 contained two HAPl-binding sites that direct activation via the acidic activation domain of HAP1. Deletion of this domain in the HAP1 $\Delta \mathrm{Kp}$ construct (or HAP1-18 $\mathrm{Kp}$ ) reduced activity to background levels (Table 1, column one). Transformants were transferred on filters to X-gal plates with galactose to induce expression of HAP1. Colonies with a reduced or an increased level of $\beta$-galactosidase were chosen for analysis. The HAPl plasmids from these candidates were recovered in bacteria and retransformed into yeast strains bearing each of the reporters.

\section{Classes of mutants recovered}

The levels of $\beta$-galactosidase in yeast cells retransformed with mutant candidates were assayed (Table 1). Mutants fell into two main categories: Class 1 showed a reduced level of activity at both UAS1 and CYC7 (Table 1, column one); class 2 mutants were fully active at UAS1 but totally inactive at CYC7 (Table 1, column one). The reciprocal class, which would reduce activity at UAS1 but not CYC7, was not observed (with the exception of the HAP1-18 mutation, see below). Mutations of both classes were sequenced. Many class 1 mutations resulted in chain termination and are not shown in Table 1. The class 1 and class 2 mutants described below all bore missense mutations.

We wished to test the possibility that class 1 mutants had a reduced binding affinity for both UAS1 and CYC7, whereas class 2 mutants had lost the ability to bind to CYC7 but not UAS1. For this purpose, gel-shift analysis was carried out in yeast extracts from cells that were partially induced for HAP1 synthesis in $1 \%$ galactose plus $1 \%$ glucose (Fig. 2). HAPl and HAP1-18 binding were also measured as controls.

Many mutants did display a reduction in DNA binding, which could account for their loss in activation ability [R57H, K76T (Fig. 2); K71N, K76R, C81Y, K86I, and FS91 (data not shown)]. Unexpectedly, some mutants in both class 1 and class 2 displayed a level of DNA binding approximately equal to or greater than the wild-type protein $(\geqslant 90 \%$ of the wild-type-binding activity). The quantitation of the binding data by the PhosphorImager is

Table 1. In vivo activation and in vitro binding of wild-type and mutant HAP1

\begin{tabular}{|c|c|c|c|c|c|}
\hline \multirow[b]{2}{*}{ Plasmid } & \multirow[b]{2}{*}{ Mutation } & \multicolumn{2}{|c|}{$\begin{array}{l}\text { In vivo activation } \\
(\beta \text {-gal units })\end{array}$} & \multicolumn{2}{|c|}{$\begin{array}{l}\text { In vitro binding } \\
\text { (\% wild type) }\end{array}$} \\
\hline & & CYC1 & CYC7 & CYC1 & CYC7 \\
\hline HAP1 & Wild type & 68 & 21 & 100 & 100 \\
\hline $\mathrm{HAP} 1 \Delta \mathrm{Kp}$ & $\Delta 1307-1483$ & 5.0 & 0.2 & - & - \\
\hline HAP $1-18$ & Ser-63-Arg & 2.3 & 264 & 3 & 50 \\
\hline HAP1-18 $\Delta \mathrm{Kp}$ & $\Delta 1307-1483$ & 2.2 & 0.5 & - & - \\
\hline \multicolumn{6}{|l|}{ Class 1} \\
\hline R57H & Arg-57-His & 10 & 0.7 & 40 & 15 \\
\hline PC4 & Pro-61-Gln & 18 & 0.6 & 150 & 95 \\
\hline $\mathrm{K} 76 \mathrm{~T}$ & Lys-76-Thr & 12 & 0.5 & 80 & 30 \\
\hline P79R & Pro-79-Arg & 17 & 0.3 & - & - \\
\hline PC5 & His-94-Asp & 18 & 0.4 & 75 & 90 \\
\hline PC6 & Thr-127-Ile Leu-128-Arg & 40 & 0.9 & 60 & 105 \\
\hline PC7 & Ser-63-Gly & 3.7 & 0.5 & $<1$ & 170 \\
\hline $\mathrm{K} 71 \mathrm{~N}$ & Lys-71-Asn & 5.3 & 0.6 & $<1$ & $<1$ \\
\hline $\mathrm{K} 76 \mathrm{R}$ & Lys-76-Arg & 5.2 & 0.5 & $<1$ & $<1$ \\
\hline C81Y & Cys-81-Tyr & 5.8 & 0.7 & $<1$ & $<1$ \\
\hline K86I & Lys-86-Ile & 2.0 & 1.2 & $<1$ & $<1$ \\
\hline \multicolumn{6}{|l|}{ Class 2} \\
\hline $\mathrm{PCl}$ & Lys-54-Glu & 55 & 0.4 & 290 & 270 \\
\hline $\mathrm{PC} 2$ & Arg-55-Gly & 257 & 0.8 & 415 & 385 \\
\hline DM1 & Pro-61-Ala Ser-63-Cys & 84 & 0.2 & 60 & 40 \\
\hline I66S & Ile-66-Ser & 108 & 0.3 & 20 & 10 \\
\hline PC3 & Gln-98-Leu Ala-101-Glu & 69 & 0.6 & 105 & 90 \\
\hline
\end{tabular}

Mutations or deletions of HAPl mutants are given in the two left columns. In vivo activity was determined by measuring $\beta$-galactosidase levels produced from reporters pLG $312 \Delta \mathrm{AX}-H I S$ (CYC1) or pCYC7-5-HIS4 (CYC7) as described in Material and methods. In vitro binding was measured from the gel shown in Fig. 2 using a PhosphorImager (Molecular Dynamics). All data were obtained under moderate levels of HAP1 using a $1 \%$ glucose-1\% galactose mix. Deletion of amino acids $1307-1483$ removes the acidic activation domain. 


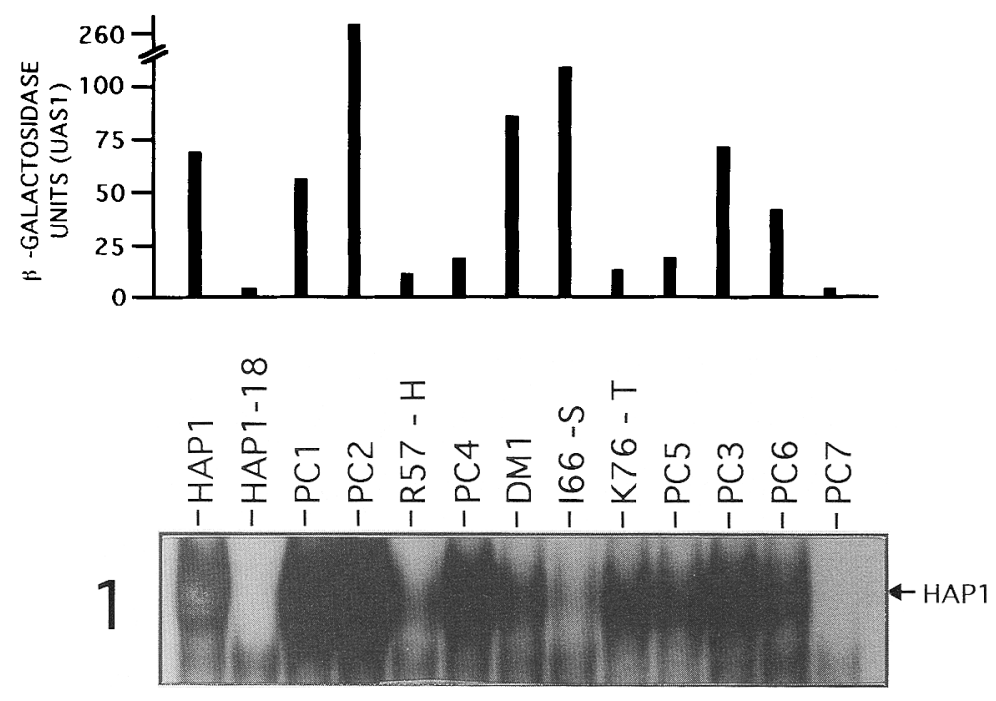

Figure 2. In vitro binding of wild-type and HAP1 mutants. (Middle) Extracts from cells expressing wildtype and HAPl mutants were prepared as described in Materials and methods and used for gel-shift assay using a probe corresponding to the UAS1 of CYC1 (1) or the UAS from $C Y C 7$ (7). With the CYCl probe, a nonspecific signal with a faster mobility than HAP1 was also detected. This nonspecific signal was similar in all lanes, indicating that no general protein degradation occurred during extract preparation and that similar amounts of protein were loaded in each lane. Shown are histograms giving the in vivo activity of the mutants using the CYC1 (top) or the CYC7 reporter (bottom). Histograms are derived from data presented in Table 1.
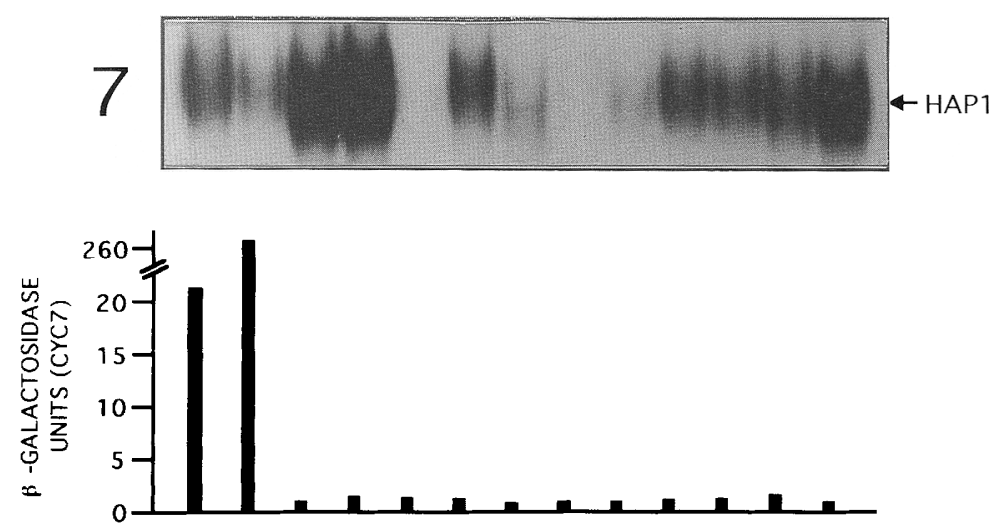

shown in Table 1. Mutants that can still bind to their DNA sites but are unable to activate transcription were identified in the $\lambda$ repressor (Guarente et al. 1982b; Hochschild et al. 1983) and in the glucocorticoid receptor (Schena et al. 1989) and termed PC mutants. In addition, mutations in Oct-1 were identified that prevented interaction with VP16 but did not prevent DNA binding (Stern and Herr 1991). It is inferred that PC mutants have lost the ability to make a protein contact with the transcriptional machinery that is critical to the activation process.

Some positive-control mutants displayed the PC property at both UAS1 and CYC7, that is, they were class 1 mutants (PC4, PC5, and PC6). One other mutant, PC7, displayed the PC property at $\mathrm{CYC7}$ and was unable to bind to UAS1. This change is of particular interest because it occurs at the $1-18$ position altering Ser-63 to Gly. Strikingly, PC1, PC2, and PC3 behaved like wildtype HAPl at UASl in terms of binding and activation but were PC mutants at CYC7. These PC mutants provide insight into the mechanism of HAP1 activation of transcription, as we discuss below.

A final class of mutant had elevated activity at CYC7 (the HAP1-18 phenotype). DNA sequence analysis of three independent mutants of this class revealed three different nucleotide alterations to change Ser-63 to Arg (data not shown). Evidently, the only change in the pro- tein that gives a level of expression at CYC7 that is elevated over wild type is Ser-63 to Arg. This is consistent with earlier findings that two different HAP1-18-type mutations changed Ser-63 to Arg (Creusot et al. 1988; Pfeifer et al. 1989).

The location of the PC mutations in HAP1 is striking. They all lie in the regions immediately to the amino or carboxyl side of the cysteine cluster of HAPl (Fig. 3). Mutations in positions within the cysteine cluster invariably affect DNA binding to both UAS1 and CYC7. Position 63 appears to be unique in differentially affecting binding to one site (UAS1) but not to the other (CYC7). Previous work indicated that serine, threonine, or cysteine residues were required at position 63 for binding to UAS1, whereas these and many other residues allowed binding at CYC7 (Kim and Guarente 1989). This work furthers our understanding of the importance of position 63 in activation at CYC7; Ser-63 gives a moderate level of transcriptional activation at CYC7, Arg-63 displays an accentuated level of activation, and Gly-63 does not activate.

\section{HAP1 activity in a gall1 deletion strain}

GAL11 is thought to aid activity of GAL4 and PPR1 by interacting in some way with the zinc finger (Himmel- 


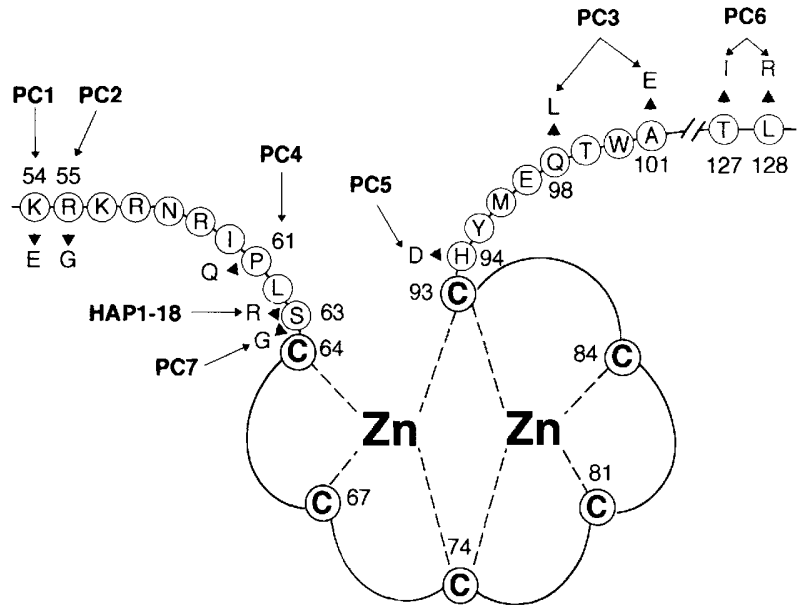

Figure 3. Positions and amino acid changes of HAP1 mutants that bind to UAS1 and CYC7 but show a reduced or no transcriptional activity (PC mutants). Circles represent amino acids; numbers represent their respective positions. Arrows indicate the mutations. The HAP1 zinc finger was drawn assuming that it has a structure similar to GAL4 (Pan and Coleman 1990). Cysteine residues involved in zinc binding are shown in boldface type.

farb et al. 1990). Because the zinc fingers of GAL4 and HAP1 are very similar (43\% identical), it was possible that GAL11 also modulated the activity of HAP1. The PC mutants could be defective in such an interaction. To test the effect of GAL11 on HAP1 activity, we deleted GAL11 from LPY22 ( $\Delta$ hap 1). The chromosomal deletion removes the promoter and most of the coding region of GAL11. We then introduced into the GAL11 or gal11 strains plasmids expressing HAP1, HAP1-PC3, or HAP118 under the control of the HAP1 promoter along with a reporter plasmid, with the lacZ gene driven by UAS1 or CYC7. In control experiments, we found that the level of a GAL4-driven reporter, pLGSD5 (Guarente et al. 1982a), was reduced 80-fold in the gal11 mutant, whereas the activity of a reporter driven by LexA-GCN4 (Hope and Struhl 1986) was unaffected in the gal11 strain (Table 2). HAP1 activity at UAS1 was reduced only about threefold by the gal11 deletion, and activity at CYC7 was reduced less than twofold. Activity of PC3 at UAS1 was affected to a similar extent as wild-type HAP1. From these results, we conclude that GAL11 plays at best a minor role in modulating HAP1 activity at both UAS1 and CYC7. Similarly, HAP1-18 activity at CYC7 was reduced only twofold in the gal11 mutant (Table 2). This finding indicates that the increase in $\mathrm{CYC} 7$ expression caused by the HAP1-18 mutation is not due to a favored interaction with GAL11.

\section{Discussion}

\section{Multisequence recognition: DNA binding}

Previous work showed that HAP1 bound primarily to one face of the DNA helix at both UAS1 and CYC7 and that major groove contacts were dispersed over three turns of the helix at both sites (Pfeifer et al. 1987b). Furthermore, at both sites, the major groove contacts were spread over a 17-nucleotide region and HAP1 made a single contact on the reverse face of the helix at one end of each site. Recent experiments indicate that HAP1 binds to these sites as a dimer (L. Zhang and L. Guarente, unpubl.).

The saturation mutagenesis of the HAP1 DNA-binding domain could potentially provide insight by identifying mutants that selectively bound to UAS1 or CYC7. However, the only changes that significantly affected binding selectively were at Ser-63, a position already known to be critical for binding to UAS1 but not CYC7. This suggests that the same set of amino acid residues of HAP1, except Ser-63, are important for binding to both sites. Ser-63 is uniquely important for binding to UAS1. In this regard, one primary difference in major groove contacts at UAS1 and CYC7 is two additional contacts at UAS1, at G262, and on the opposite strand at G252 (Pfeifer et al. 1987b). These residues are 10 bases apart and are therefore on the same face of the helix. We speculate that Ser-63 of two monomers of HAP1 directly contacts G262 and G252 in UAS1.

\section{Multisequence recognition: site-specific cofactors}

In studying what biological role may be served by evolution of two HAP1-binding sites of different sequence, we have reported differences in the levels of activation and extent of regulation between UASI and CYC7 (Kim and Guarente 1989; L. Guarente, unpubl.). Furthermore, past work showed that certain derivatives of HAP1 bear-

Table 2. Effect of GAL11 deletion on HAP1 activity at CYC1 (reporter pLGA312 $\triangle A X-\mathrm{HIS}$ and CYC7 (reporter $p$ CYC7-5-HIS4)

\begin{tabular}{llrr}
\hline & & \multicolumn{2}{c}{ Strain } \\
\cline { 3 - 4 } Reporter & Activator & wild type & gal11 \\
\hline CYC1 & HAP1 & 3,045 & 930 \\
& HAP1-18 & 25 & 60 \\
& HAP1-PC3 & 4,200 & 1410 \\
& - & 20 & 40 \\
CYC7 & HAP1 & 740 & 475 \\
& HAP1-18 & 6,720 & 3210 \\
& HAP1-PC3 & 15 & NT \\
pLGSD5 & - & 35 & 20 \\
LexA (RB115) & GAL4 & 25,000 & 320 \\
\hline
\end{tabular}

HAP1 expression vectors are under the control of their own promoter (see Material and methods), and YEP352 (Hill et al. 1986) was used in the - HAP control. In vivo activity was determined by measuring $\beta$-galactosidase activity, as described in Material and methods, using a wild-type (LPY22) or a gal11 (LPgal11 ${ }^{-}$) strain. $\beta$-Galactosidase units are in nmoles/min per $\mathrm{mg}$ of protein. (NT) Not tested. 
ing internal deletions bound normally to both sites but were specifically defective in activation at CYC7 (Kim et al. 1990). These findings suggested that there was some difference in activation by HAP1 at UAS1 versus CYC7.

What might this difference be? A very interesting type of mutation that emerged from our screen bound normally to both UAS1 and CYC7 but were defective in activation of transcription. One class of these PC mutants was defective in activation at both UAS1 and CYC7, whereas a second class activated like wild type at UAS1 but was inactive at CYC7. All of these PC mutations were in residues that flank the 6 cysteine residues of the zinc finger (Fig. 3). These PC mutations lie $>1200$ residues away from the carboxyl acidic activation domain of HAPl.

From these findings we surmise that the DNA-binding domain of HAPl contains specificity to interact with another protein factor, or cofactor, most probably by cobinding with that factor at HAPl sites in vivo. Recently, the three-dimensional structure of the DNAbinding domain of GAL4 bound to DNA has been determined (Marmorstein et al. 1992). GAL4 binds as a dimer to its recognition sequence with a twofold symmetry. Interestingly, contacts with DNA are located at both ends of the UAS leaving a long stretch of DNA at the center accessible for binding of another protein in the major groove. Assuming that HAPl has a structure similar to GAL4, the residues altered by $P C 1, P C 2, P C 3$, $P C 4, P C 5$, and $P C 7$ mutations could be in a position to interact with a cofactor bound to the center of the site (Fig. 4, model 1). Protein-protein interactions of this type have been observed in the case of the yeast repressor

\section{MODEL 1}
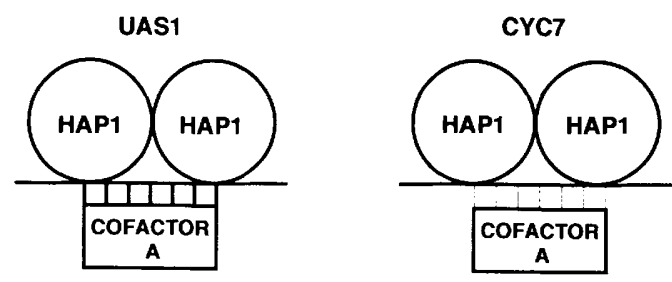

MODEL 2
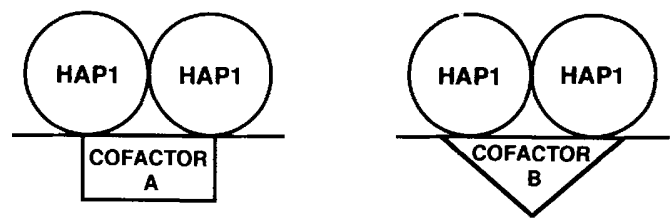

Figure 4. Models for interaction between HAPl and cofactor(s). HAP1 is shown bound to UASI or CYC7 as a dimer. In model 1 , only one cofactor interacts with the DNA-binding domain of HAP1 and a UAS. Thick stripes represent a strong interaction with the HAP1-DNA complex at UAS1; thin stripes represent a weaker interaction at CYC7. In model 2, two different cofactors, each specific for a given UAS, are responsible for the differential activity at UAS1 and CYC7. $\alpha 2$ and the factor MCM1 (Keleher et al. 1988). The PC6 double mutant lies in more distal residues that are important in dimerization (L. Zhang and L. Guarente, unpubl.) and may alter the conformation of the DNA-binding domain of $\mathrm{HAP} 1$, resulting in a loss of interaction with a cofactor when bound at CYC7. We note that the PC mutations do not alter the complex between HAP1 in yeast extracts and its binding sites, suggesting that the cofactor is too unstable or its affinity for the HAP1DNA complex is too low for detection by gel shift.

Perhaps the most intriguing class of mutations, exemplified by $P C 1, P C 2$, and $P C 3$, behave like the wild-type protein at UAS1 and are PC mutants at CYC7. How can we explain these effects? One reasonable model is that HAP1 associates with two different cofactors that are specific for UAS1 and CYC7 (Fig. 4, model 2). This specificity could be determined by the difference in sequences of the two sites. Different cofactors might also explain why UAS1 activity is higher than that of CYC7 even though HAP1 binds to the sites with the same affinity (Kim and Guarente 1989). The PC1, PC2, and PC3 changes would selectively knock out the interaction with the CYC7 cofactor by this model. However, this class of mutants do not eliminate model 1 , if we assume that the cofactor-DNA interaction at UAS1 is stronger than at $\mathrm{CYC7}$ and can compensate for the loss of interaction energy caused by $P C 1, P C 2$, and PC3. It will be necessary to identify the gene encoding the $\mathrm{CYC7}$ cofactor to clearly discriminate between the two cofactor models.

We tested the possibility that GAL11 was a HAP1 cofactor because it affects the activity of GAL4 and shows specificity for the DNA-binding domain of GAL4, which is similar to that of HAP1. However, removing GAL11 from cells by gene deletion exerted a minor reduction in the activity of UASl (threefold) and had a slight effect at CYC7 (less than twofold), indicating that GAL11 is not an important cofactor for HAP1.

One HAP1 mutant that is striking in its effects is HAP1-18, changing Ser-63 to Arg, which elevates HAP1 activity at CYC7 10-fold or more. The mutation does not increase the steady-state level of HAP1 nor the affinity of HAP1 for CYC7 (Kim and Guarente 1989). We have speculated that HAP1-18 increases the affinity of HAP1 for some component of the transcriptional machinery, which we now envision as a cofactor at CYC7. The change of Ser-63 to Gly (PC7) has the opposite effect of HAP1-18. The mutant PC7 is inactive at CYC7, although it still binds to DNA. We expect this change to disrupt interaction with a CYC7 cofactor. In a previous study (Kim and Guarente 1989), we found that the change from Ser-63 to Gly displayed somewhat reduced binding when synthesized in vitro and partial activation potential in vivo at CYC7 (Kim and Guarente 1989). In that study, the CYC7 reporter contained the entire CYC7 promoter and was integrated into the yeast genome. Apparently, the CYC7 reporter used here bearing only the HAP1-binding site oligonucleotide provides a more stringent assay for HAPl activation.

This study (Table 1) and previous experiments have 
shown that the acidic domain at the carboxy-terminal part of HAP1 is necessary for activation while the PC mutants indicate that the activation domain is not sufficient for activation of transcription. One possibility to account for these observations is that the cofactor acts in synergy with the acidic domain to stabilize an interaction with a target protein of the transcriptional machinery. Alternatively, the cofactor could help to stabilize occupancy of the UAS by HAP1 in vivo.

In summary, our mutational analysis of the DNAbinding domain of HAP1 has provided insights into DNA binding and transcriptional activation at dissimilar UASs. Most novel is the surmise that HAPl uses a cofactor at CYC7 and, potentially, a different cofactor at UAS1. The difference in sequences of the two sites could specify which cofactor is bound. The genetic approach illustrated here may be an effective route to begin to identify cofactors for other activators.

\section{Materials and methods}

Strains and media

Strains are derivatives of BWG1-7A (Guarente and Mason 1983). LPY22 contains a LEU2 deletional insertion in HAP1 between the HindIII and the KpnI sites of HAP1. This totally removes HAP1 functional sequences. LPY22 was rendered trp $1^{-}$by integrating the URA3-hisG cassette (Alani et al. 1987) into the TRP1 gene and selecting for URA ${ }^{+}$and subsequently gal11 ${ }^{-}$ (Himmelfarb et al. 1990). This disruption replaces the GAL11 sequences from $123 \mathrm{bp}$ upstream of the ATG to nucleotide +2341 by the TRP1 gene. Southern blotting confirmed proper integration. Rich (YPD), minimal (SD), and synthetic complete (SC) media were prepared as described (Sherman et al. 1986).

\section{Plasmids}

Plasmid pEMBL-HAP1 was constructed by subcloning the 1.3$\mathrm{kb}$ BamHI fragment (encoding the amino-terminal part of HAP1) from SD5-HAP1 (Kim and Guarente 1989) into the BamHI site of pEMBL18 + (Dente et al. 1985). An Xhol site was then introduced by site-directed mutagenesis at nucleotide 111 relative to the ATG using the oligonucleotide 5' GCCAACACCTCGAGTAATCT- $3^{\prime}$ to give the plasmid pEMBL-HAP1CAS. Introduction of the $X h o I$ site did not change the HAPl amino acid sequence. SD5-HAP1-CAS was constructed in two steps. The unique Xhol site of SD5-HAP1 in the CYC1 promoter was destroyed by cutting the plasmid with XhoI, filling in with Klenow, and ligating. The resulting plasmid was then used to replace its $B a m H I$ fragment by the one from pEMBL-HAP1CAS, thus giving a plasmid with an XhoI-BstEII /nucleotide +739 relative to the ATG) cassette fragment.

Deletion mutants SD5-HAP1 $\triangle K p$ and SD5-HAP1-18DKp were constructed by linearizing the plasmids SD5-HAP1 and SD5-HAP1-18 (Kim and Guarente 1989) by KpnI, filling in with Klenow, and inserting an $\mathrm{XbaI}$ linker (New England Biolabs) containing nonsense codons in the three reading frames. HAP1 expression vector under the control of its own promoter was constructed in two steps. The AatII-Ncol fragment (containing a $2 \mu$ origin of replication) was isolated from plasmid YEP352 (Hill et al. 1986) and subcloned into the AatII-NcoI backbone of plasmid pRS306 (Sikorski and Hieter 1989), giving p306-2 $\mu$ containing a unique $P s t \mathrm{I}$ and $X$ hol sites in the polylinker region. The 5.3-kb PstI-XhoI fragment containing the HAP1 gene (from
-227 to +5013 bp relative to the AUG) was isolated from plasmids pHAP1 (Pfeifer et al. 1987b) and subcloned into the XhoIPstI backbone of $\mathrm{p} 306-2 \mu$ to give pHAP1-S. HAP1 mutations in plasmids pHAP1-18 (Pfeifer et al. 1989) and PC3 (Table 1) were transferred to the expression vector pHAP1-S by replacing their corresponding EcoRI fragments |+176 to +602 relative to the ATG) to give pHAP1-18-S and pHAP1-PC3, respectively (Table 2).

The CYC1 reporter, pLG $\Delta 312 \Delta \mathrm{AX}-H I S$, was constructed by removing the URA3 marker of plasmid pLG $\triangle 312 \Delta \mathrm{AX}$ (Guarente et al. 1984) by digesting with StuI and inserting a blunt-ended SacI-BstEII genomic fragment from the HIS4 gene (Donahue et al. 1982). The CYC7 reporter, CYC7-5-HIS4, was constructed by removing the URA 3 marker from plasmid CYC7-5, containing two copies of the HAP1-binding site at CYC7 (Kim and Guarente 1989), by digesting with StuI, adding NotI linkers, and inserting a blunt-ended SacI-BstEII fragment of the HIS4 gene (Donahue et al. 1982) flanked by NotI linkers.

Saturation mutagenesis of the DNA-binding domain of HAP1

Single-stranded DNA from plasmid pEMBL-HAPl-CAS was prepared (Sambrook et al. 1989) and used for saturation mutagenesis with spiked oligonucleotides (level of contamination, $2.5 \%$ for each of three other nucleotides) (see Fig. 1). Mutagenesis was performed using the phosphorothionate-modified DNA method, according to Nakamaye and Eckstein (1986), with a kit from Amersham following the manufacturer's protocol. In vitro-mutated DNA was then transformed into $E$. coli (TG1). Greater than $10^{4}$ independent colonies where then scraped off the plates and grown for plasmid isolation by cesium chloride banding (Sambrook et al. 1989). Mutated fragments where then excised from pEMBL-HAP1-CAS by cutting with Xhol (at position +111 relative to the ATG) and BstEII $(+739)$ for subcloning into the XhoI-BstEII backbone of the yeast expression vector SD5-HAP1-CAS. The latter contained the URA3 marker and a $2 \mu$ origin of replication. The ligation mix was then transformed into E. coli MC1061, and the colonies were pooled for plasmid isolation. The DNA was then used to transform the yeast strain LPY22 bearing a HIS4-marked reporter (see above) on minimal plates. Colonies were then transferred with a nitrocellulose filter onto a minimal X-gal plate containing $2 \%$ galactose. Colonies with altered $\beta$-galactosidase activity relative to wild type were then grown for plasmid isolation and transformation into $E$. coli. Mutations were identified by sequencing the double-stranded DNA (Sanger et al. 1977). Mutant phenotypes were confirmed by transforming them back into the LPY22 strain with either the CYC1 or the $C Y C 7$ reporters.

\section{$\beta$-Galactosidase assays}

HAP1 expression vectors under the control of UAS $S_{\mathrm{GAL}}$ Cells were grown to saturation in minimal media containing $2 \%$ raffinose; they were then diluted 20 times in SD media containing $1 \%$ glucose and $1 \%$ galactose and grown for $\sim 18 \mathrm{hr}$ before assaying for $\beta$-galactosidase (Table 1; Guarente and Mason 1983).

gall1- strain Cells were grown to saturation in YPD; they were then spun, washed in water, and diluted 2000 times (LPY22 strain) or 50 times (LPgall 1- strain) in SC media with $2 \%$ glucose lacking histidine for $2 \%$ galactose plus histidine for the reporter pLGSD5). Cells were then grown for $\sim 20 \mathrm{hr}$ before assaying for $\beta$-galactosidase activity (Table 2; Rose and Botstein 1983).

Preparation of extracts and DNA-binding assays

Cells were grown exactly as described for HAPl expression vec- 
tors under the control of $\mathrm{UAS}_{G A L}$. Extracts were then prepared, and the DNA-binding assay was carried out as described (Pfeifer et al. 1987a) using complementary oligonucleotides with XhoI ends filled in with the Klenow fragment and $\left[\alpha^{-32} \mathrm{P}\right] \mathrm{dATP}$ and $\left[\alpha^{-32} \mathrm{P}\right] \mathrm{dGTP}$. The sequence of the CYC1 probe is $5^{\prime}-\mathrm{TC}$ GAGTGGCCGGGGTTTACGGACGATGA-3', and that of the CYC7 probe is 5'-TCGACCCTCGCTATTATCGCTATTAGC$3^{\prime}$. Quantitative data were obtained by measuring the amount of radioactivity present in the retarded bands using a PhosphorImager (Molecular Dynamics).

\section{Acknowledgments}

We are grateful to Howard Himmelfarb (Harvard University, Cambridge, MA) for advice and the gift of plasmids. We thank J. Fikes and P. Sugiono for plasmids and B. Pina, N. Silverman, and L. Zhang for critical reading of the manuscript. This work was supported by the National Institutes of Health (grant GM30454) to L.G. B.T. is supported by a Centennial postdoctoral fellowship from the Medical Research Council of Canada.

The publication costs of this article were defrayed in part by payment of page charges. This article must therefore be hereby marked "advertisement" in accordance with 18 USC section 1734 solely to indicate this fact.

\section{References}

Alani, E., L. Cao, and N. Kleckner. 1987. A method for gene disruption that allows repeated use of URA3 selection in the construction of multiply disrupted yeast strains. Genetics 116: $541-545$.

Banerii, J., S. Rusconi, and W. Schaffner. 1981. Expression of a beta-globin gene is enhanced by remote SV40 DNA sequences. Cell 27: 299-308.

Brent, R. and M. Ptashne. 1985. A eukaryotic transcriptional activator bearing the DNA specificity of a prokaryotic repressor. Cell 43: 729-736.

Clavilier, L., G. Péré-Aubert, M. Somlo, and P.P. Slonimski. 1976. Réseau d'interactions entre des gènes non liés: Régulation synergique ou antagoniste de la synthèse de l'iso-1cytochrome $c$, de l'iso-2-cytochrome $c$ et du cytochrome $b_{2}$. Biochimie 58: 155-171.

Creusot, F., J. Verdière, M. Gaisne, and P.P. Slonimski. 1988. CYP1 (HAP1) regulator of oxygen-dependent gene expression in yeast. I. Mol. Biol. 204: 263-276.

Dente, L., M. Sollazzo, C. Baldari, G. Cesareni, and R. Cortese. 1985. The pEMBL family of single-stranded vectors. In DNA cloning (ed. D.M. Glover), vol. I, pp. 101-107. IRL Press, London, UK.

Donahue, T.F., P.J. Farabaugh, and G.R. Fink. 1982. The nucleotide sequence of the HIS4 region of yeast. Gene 18: 47-59.

Guarente, L. and O. Bermingham-McDonogh. 1992. Conservation and evolution of transcriptional mechanisms in eukaryotes. Trends Genet. 8: 27-32.

Guarente, L. and T. Mason. 1983. Heme regulates transcription of the CYC1 gene of $S$. cerevisiae via an upstream activation site. Cell 32: 1279-1286.

Guarente, L., R.R. Yocum, and P. Gilford. 1982a. A GAL10$\mathrm{CYCl}$ hybrid yeast promoter identifies the GAL4 regulatory region as an upstream site. Proc. Natl. Acad. Sci. 79: 74107414.

Guarente, L., J.S. Nye, A. Hochschild, and M. Ptashne. 1982b. A mutant $\lambda$ repressor with a specific defect in its positive control function. Proc. Nat1. Acad. Sci. 73: 2249-2253.

Guarente, L., B. Lalonde, P. Gifford, and E. Alani. 1984. Dis- tinctly regulated tandem upstream activation sites mediate catabolite repression of the CYC1 gene of $S$. cerevisae. Cell 36: $503-511$.

Hill, J.E., A.M. Myers, T.J. Koerner, and A. Tzagoloff. 1986. Yeast/E. coli shuttle vectors with multiple unique restriction sites. Yeast 2: 163-167.

Himmelfarb, H., J. Pearlberg, D.H. Last, and M. Ptashne. 1990. GAL11P: A yeast mutation that potentiates the effect of weak GAL4-derived activators. Cell 63: 1299-1309.

Hochschild, A., N. Irwin, and M. Ptashne. 1983. Repressor structure and the mechanism of positive control. Cell 32: 319-325.

Hope, I.A. and K. Struhl. 1986. Functional dissection of a eukaryotic transcriptional activator protein, GCN4 of yeast. Cell 46: 885-894.

Jarvis, E.E., K.L. Clark, and G.F. Sprague. 1989. The yeast transcription activator PRTF, a homolog of the mammalian serum response factor, is encoded by the MCM1 gene. Genes \& Dev. 3: 936-945.

Keleher, C.A., C. Goutte, and A.D. Johnson. 1988. The yeast cell-type-specific repressor $\alpha 2$ acts cooperatively with a non cell-type-specific protein. Cell 53: 927-936.

Kim, K.S. and L. Guarente. 1989. Mutations that alter transcriptional activation but not DNA binding in the zinc finger of yeast activator HAP1. Nature 342: 200-203.

Kim, K.S., K. Pfeifer, L. Powell, and L. Guarente. 1990. Internal deletions in the yeast transcriptional activator HAPl have opposite effects at two sequence elements. Proc. Natl. Acad. Sci. 87: 4524-4528.

Ma, J. and M. Ptashne. 1987. Deletion analysis of GAL4 defines two transcriptional activating segments. Cell 48: 847-853.

Marmorstein, R., M. Carey, M. Ptashne, and S. Harrisson. 1992. DNA recognition by GAL4: Structure of a protein-DNA complex. Nature 356: 408-414.

Moreau, P., R. Hen, B. Wasylyk, R. Everett, M.P. Gaub, and P. Chambon. 1981. The SV40 72 base pair repeat has a striking effect on gene expression both in SV40 and other chimeric recombinants. Nucleic Acids Res. 9: 6047-6056.

Nakamaye, K. and F. Eckstein. 1986. Inhibition of restriction endonuclease Ncil cleavage by phosphorothionate groups and its application to oligonucleotide-directed mutagenesis. Nucleic Acids Res. 14: 9679-9698.

Pan, T. and J.E. Coleman. 1990. GAL4 transcription factor is not a "zinc finger" but forms a $\mathrm{Zn}(\mathrm{II})_{2} \mathrm{Cys}_{6}$ binuclear cluster. Proc. Natl. Acad. Sci. 87: 2077-2081.

Passmore, S., R. Elble, C. Christ, and B.K. Tye. 1989. A protein involved in minichromosome maintenance in yeast binds a transcriptional enhancer conserved in eukaryotes. Genes \& Dev. 3: 921-935.

Pfeifer, K., B. Arcangioli, and L. Guarente. 1987a. Yeast HAP1 activator competes with the factor $\mathrm{RC} 2$ for binding to the upstream activation site UAS1 of the CYC1 gene. Cell 49: 918.

Pfeifer, K., T. Prezant, and L. Guarente. 1987b. Yeast HAP1 activator binds to two upstream activation sites of different sequence. Cell 49: 19-27.

Pfeifer, K., K.S. Kim, S. Kogan, and L. Guarente. 1989. Functional dissection and sequence of yeast HAP1 activator. Cell 56: 291-301.

Prezant, T., K. Pfeifer, and L. Guarente. 1987. Organization of the regulatory region of the yeast $C Y C 7$ gene: Multiple factors are involved in regulation. Mol. Cell. Biol. 7: 32523259.

Rose, M. and D. Botstein. 1983. Structure and function of the yeast URA3 gene: Differentially regulated expression of hybrid $\beta$-gal from overlapping coding sequences in yeast. $/$. Mol. 
Biol. 170: 833-904.

Sambrook, J., E.F. Fritsch, and T. Maniatis. 1989. Molecular cloning: A laboratory manual. Cold Spring Harbor Laboratory Press, Cold Spring Harbor, New York.

Sanger, F., S. Nicklen, and A.R.Coulson. 1977. DNA sequencing with chain-terminating inhibitors. Proc. Natl. Acad. Sci. 74: 5463-5467.

Schena, M., L.P. Freedman, and K.R. Yamamoto. 1989. Mutations in the glucocorticoid receptor zinc finger region that distinguish interdigitated DNA binding and transcriptional enhancement activities. Genes \& Dev. 3: 1590-1601.

Schneider, J.C. and L. Guarente. 1991. Regulation of the yeast CYT1 gene encoding cytochrome $c 1$ by HAP1 and HAP2/3/ 4. Mol. Cell. Biol. 11: 4934-4942.

Sherman, F., G.R. Fink, and J.B. Hicks. 1986. Methods in yeast genetics. Cold Spring Harbor Laboratory, Cold Spring Harbor, New York.

Sikorski, R.S. and P. Hieter. 1989. A system of shuttle vectors and yeast host strains designed for efficient manipulation of DNA in Saccharomyces cerevisae. Genetics 122: 19-27.

Stern, S. and W. Herr. 1991. The herpes simplex virus transactivator VP16 recognizes the Oct-1 homeo domain: Evidence for a homeo domain recognition subdomain. Genes \& Dev. 5: 2555-2566.

Suzuki, Y., Y. Nogi, A. Abe, and T. Fukasawa. 1988. GAL11 protein, an auxiliary transcription activator for genes encoding galactose-metabolizing enzymes in Saccharomyces cerevisae. Mol. Cell. Biol. 8: 4991-4999.

Winkler, H., G. Adam, E. Mattes, M. Schanz, A. Hartig, and H. Ruis. 1988. Co-ordinate control of synthesis of mitochondrial genes and non-mitochondrial hemoproteins: A binding site for the HAP1 (CYP1) protein in the UAS region of the yeast catalase T gene (CTT1). EMBO J. 7: 1799-1804.

Zitomer, R.S., J.W. Sellers, D.W. McCarter, G. A. Hastings, P. Wick, and C. Lowry. 1987. Elements involved in oxygen regulation of the Saccharomyces cerevisiae CYC7 gene. Mol. Cell. Biol. 7: 2212-2220. 


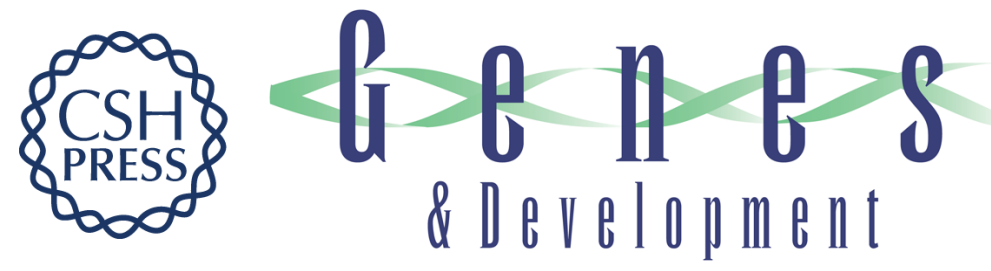

\section{HAP1 positive control mutants specific for one of two binding sites.}

B Turcotte and L Guarente

Genes Dev. 1992, 6:

Access the most recent version at doi:10.1101/gad.6.10.2001

References This article cites 38 articles, 14 of which can be accessed free at: http://genesdev.cshlp.org/content/6/10/2001.full.html\#ref-list-1

License

Email Alerting

Receive free email alerts when new articles cite this article - sign up in the box at the top Service right corner of the article or click here.

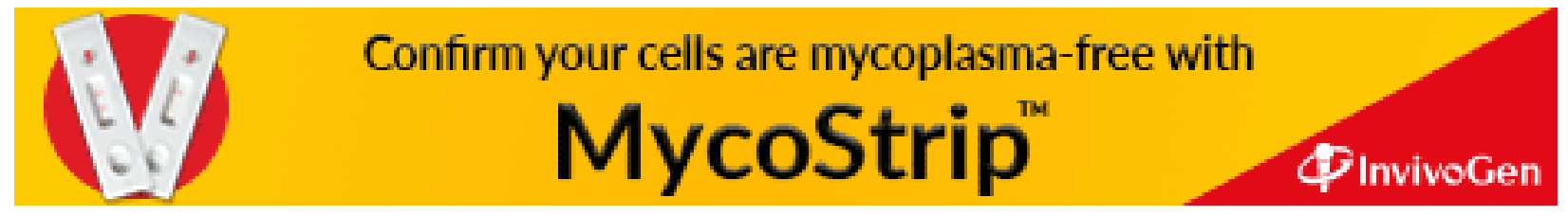

